\title{
The long-lived queen: reproduction and longevity in female eusocial Damaraland mole-rats (Fukomys damarensis)
}

\author{
Christina M. Schmidt ${ }^{*}$, Jennifer U.M. Jarvis ${ }^{2}$ \& Nigel C. Bennett ${ }^{1}$ \\ ${ }^{1}$ Department of Zoology and Entomology, University of Pretoria, Pretoria, 0001 South Africa \\ ${ }^{2}$ Department of Zoology, University of Cape Town, Rondebosch, Cape Town, 7700 South Africa
}

Received 14 March 2013. Accepted 7 May 2013

\begin{abstract}
The inverse relationship between reproduction and lifespan is one of the main concepts of life history theory. This association has been observed in most taxa, although exceptions have been found in which a breeding female outlives her non-reproductive cohorts. This relationship is well known in social insects, and it has recently come to light that reproductive females of certain social mole-rat species also exhibit extended lifespans relative to non-breeders. We analysed mark-recapture data over 13 years in 12 colonies of Damaraland mole-rats (Fukomys damarensis) to assess if colony queens lived longer than non-reproductive adult females. Queens were recaptured up to 8.5 years after initial capture $(\bar{X}=$ 6.2 years); significantly longer than non-reproductive females ( $\bar{X}=1.3$ years), suggesting that a colony queen lives longer than her non-reproductive subordinates. This difference may be attributed to both physiological and social factors which may relax reproductive costs in queens.
\end{abstract}

Key words: Fukomys damarensis, reproductive costs, sociality, longevity, trade-offs.

$O_{p o s}^{n}$ ne of the central tenets of life history theory posits that an individual produces offspring at a cost to their own survival and future reproductive success (Williams 1966; Roff 1992; Stearns 1992). Animals that reproduce early and frequently tend to have relatively short lives, and this may be, in part, due to resource partitioning; resources that would be allocated to self-maintenance are instead diverted towards the many functions involved in the production of offspring (Williams 1966; Noordwijk \& de Jong 1986; Stearns 1989; Kirkwood \& Rose 1991; Stearns 1992). Reproduction may also take its toll directly on an animal's survivorship by decreasing immune function, increasing oxidative stress, or impairing other physiological functions (Ricklefs \& Wikelski 2002; Speakman 2008). Supporting this hypothesis, the relaxation of the physiological demands of reproduction by inhibiting or reducing reproductive

*Author for correspondence. E-mail: ossavolatus@gmail.com effort can result in an increase in lifespan. Ovariectomized brown anole lizards (Anolis sagrei) and eastern lubber grasshoppers (Romalea microptera) exhibit increased survival relative to sham ovariectomized females (Hatle et al. 2008; Cox \& Calsbeek 2010), and decreased reproduction increases lifespan in Drosophila (e.g. Flatt 2011). However, many social insects show a converse effect. In social ant, termite and some bee species, fecundity is associated with long life relative to non-reproductive colony members (Remolina \& Hughes 2008).

The lifespan of eutherian mammals is, in general, inversely associated with annual fecundity (Read \& Harvey 1989). However, it has recently been established that African mole-rats (Rodentia: Bathyergidae) include species that show a lifespan-reproduction relationship that is more akin to that of the above-mentioned social insects. This family of subterranean rodents contains both social and solitary species; social mole-rats exhibit a division of labour associated with reproductive status, in which subordinate colony members are reproductively supressed via various mechanisms and reproduction is restricted to one dominant female, i.e. the queen (Bennett \& Faulkes 2000). It is within these social species that extended lifespan in reproductive females has been observed. Reproductive female Ansell's mole-rats (Fukomys anselli) and giant mole-rats (F. mechowii) live an average of $10.23 \pm 1.09$ S.E. years whereas the mean lifespan of non-reproductive females is $4.56 \pm 0.19$ S.E. years in captivity (Dammann et al. 2011); mean survival of reproductive female Ansell's mole-rats is almost twice that of non-breeding females (Dammann \& Burda 2006). Naked mole rats (Heterocephalus glaber) exhibit no difference in maximum lifespan between breeding and nonbreeding females in captivity (Sherman \& Jarvis 2002); however, according to Braude in a personal 
communication, breeding females can live at least four times longer than non-breeding individuals in the wild (it is unclear whether the non-breeders referred to in this study include male cohorts; Buffenstein 2008).

The social structure of naked mole-rats is analogous to eusocial ant, termite and some bee species (Jarvis 1981), and it has been argued that the only other mammal that exhibits this extreme type of sociality is another bathyergid species, the Damaraland mole-rat (Fukomys damarensis; Jarvis \& Bennett 1993). Reproductive suppression in Damaraland mole-rats is due to two factors: incest avoidance and social environment (Cooney \& Bennett 2000; Bennett 2011), and subordinate females possess functionally developed ovaries, but do not ovulate (Bennett 1994; Bennett et al. 1994; Molteno \& Bennett 2000). Although some individuals disperse and found new colonies, most non-reproductive members stay in their natal colony (Jarvis \& Bennett 1993); it is estimated that $90 \%$ of the population never breed (Faulkes \& Bennett 2001).

Given this similarity in reproductive patterns to both naked mole-rats and social insects, as well as to Ansell's and giant mole-rats, it is feasible that Damaraland mole-rat queens may also live longer than non-reproductive females. We analysed mark-recapture data for 12 colonies of Damaraland mole-rats over a period of 13 years to assess relative longevity between these two reproductive groups.

Twelve colonies of mole-rats were marked and recaptured every six months from April 1988 to June 2001 over an $175000 \mathrm{~m}^{2}$ area at a farm near Dordabis, Namibia $\left(22^{\circ} 58^{\prime} \mathrm{S} 17^{\circ} 41^{\prime} \mathrm{E}\right)$. The area comprises thorn scrub acacia woodland savanna with red Kalahari arenosols that support a wide diversity of geophytes upon which the animals feed. Colonies were located by the presence of above ground mounds. Opened sections of tunnel were excavated and Hickman live-traps placed into the holes. Traps were checked every two hours for a period of 10 days, and in order to ensure that all mole-rats from a colony had been collected, the traps were left in for three days following no capture. Captured mole-rats were housed in plastic holding containers $(60 \mathrm{~cm} \times 30 \mathrm{~cm} \times 30 \mathrm{~cm})$ containing wood shavings and paper towelling, and were fed local geophytes of the genus Dipcadi along with sweet potato. The containers were cleaned out every second day until release back into their tunnel system.
All colony members were weighed and sexed, and breeding status was determined in the females; queens were characterized by prominent axillary teats and a perforate vagina. Female adult body mass, which is attained at about two years of age, exceeds $130 \mathrm{~g}$ (Bennett et al. 1991), thus adult non-breeders could be distinguished from juveniles. All animals were marked by toe-clipping and were released once the entire colony had been trapped. All subsequent recaptures were recorded to measure survival following initial capture. Many mole-rats from the area were still alive following the termination of the collection period, thus recapture data does not provide the maximum potential lifespan for this species. Toe clips were placed in 100\% ethanol to be used in genetic studies elsewhere. Duration of survival following initial capture was compared between queens and adult non-reproductive females using a WelchSatterthwaite $t$-test.

Queens were present in a colony for a significantly longer duration than non-reproductive adult females $\left(t_{11.02}=9.97, P<0.0001\right)$. Queens $(n=12)$ were recaptured 3-8.5 years after initial trapping $(\bar{X}=6.2 \pm 0.49$ S.E.), while non-reproductive adult females $(n=170)$ were recaptured $0.5-4$ years $(\bar{X}=1.3 \pm 0.023$ S.E. $)$.

Damaraland queens persisted much longer within a colony than their non-reproductive adult female counterparts, suggesting that, like other social mole-rats and insects, the queen lives much longer than subordinate, non-breeding females. This apparent reversal in expected relationship between reproduction and lifespan in eusocial animals has been previously ascribed to extrinsic and physiological factors.

The 100-fold increase in lifespan of eusocial ant queens relative to solitary species is attributed to the sheltering and protection of the queen, which limits exposure to extrinsic sources of mortality compared to non-reproductive individuals (i.e. workers; Keller \& Genoud 1997; Heinze \& Schrempf 2008). However, this scenario is unlikely in social mole-rats.

Exposure to sources of extrinsic mortality, such as predators, is relatively low for subterranean species (Jarvis \& Bennett 1991). Only about 10\% of all Damaraland mole-rats that disappear from a colony are found in a new colony, suggesting that the disappearance of about $90 \%$ of colony members may be attributed to mortality rather than dispersal (Jarvis \& Bennett 1993). While it is possible that dispersal increases risk of death, captive (i.e. 
sheltered from sources of extrinsic mortality) Ansell's and giant mole-rats exhibit the same differential survival between breeders and nonbreeders as was observed in free living Damaraland mole-rats. Thus, the disappearance of nonreproductive females from the colonies is unlikely to be primarily attributable to extrinsic mortality. The difference between maximum lifespan between queens and subordinates in wild but not captive naked mole-rat colonies suggests that extrinsic sources in the wild may impact age structure within the colony (Buffenstein 2008), though this species is more exposed to potential predators when forming mole-hills than is the Damaraland mole-rat (Jarvis \& Bennett 1991).

Non-reproductive female Damaraland mole-rats commence ovulation when removed from the presence of the queen and are introduced to a non-related male (Molteno \& Bennett 2000), and this ascension to a dominant status is associated with several physiological alterations. These include an increase in brain volume, pituitary sensitivity, reproductive hormone concentrations, and body length (Bennett et al. 1993; Bennett 1994; Bennett et al. 1994; Bennett et al. 1996; Young \& Bennett 2010; Anyan et al. 2011). Although it has yet to be explored, it is possible that some of these adjustments could be correlated with extended longevity. In Ansell's mole-rats, there is some evidence that breeding females possess elevated defences against oxidant and carbonyl stress relative to their shorter-lived, non-breeding cohorts, which may lend support to the oxidative damage theory of ageing (Dammann et al. 2012).

Insect queens have more resources available to them owing to provisioning from non-reproductive colony members (Remolina \& Hughes 2008). Therefore it has been proposed that it is unnecessary for queens to divert resources from selfmaintenance, and thus they are not exposed to physiological costs that could impair survival (Remolina \& Hughes 2008). Non-reproductive Damaraland mole-rats forage for food and maintain the burrow system, allowing the breeding individuals to expend their energy almost exclusively on reproduction (Bennett 1990), and given that the queen and dominant male are the largest individuals in a colony (Bennett \& Jarvis 1988; Lovegrove 1988), adequate food resources are most likely available to support the energy and nutrient demands of offspring production. If so, then mole-rat queens would also not need to trade off self-maintenance for reproduction.
It is yet to be determined what mechanisms underlie the extended longevity of reproductive social mole-rats. It is plausible that an interaction between reduced exposure to sources of extrinsic mortality, increased resistance to physiological costs, and elevated resource availability has enabled the evolution of long lifespans in Damaraland mole-rat queens by reducing, or perhaps even eliminating reproductive costs. Given that this relationship has been observed only in social mole-rats and insects to date, there appears to be a connection between sociality and long lifespan of reproductive individuals that warrants further exploration. Insights into the longevity of reproductive social insects will provide a strong foundation from which to further explore this unusual phenomenon in social African mole-rats. These species would also serve as an apt model for investigating epigenetic regulation of lifespan and other life history traits, since dominance is regulated by environmental factors.

We thank Mr and Mrs P. Luhl for allowing us to trap on their farm Garib in Dordabis, Namibia, and M. Griffin for helping with the processing of permits from the Namibian Nature Conservation authorities. C.M.S. acknowledges funding from the University of Pretoria, J.U.M.J. acknowledges funding from the University of Cape Town and N.C.B. acknowledges funding from the University of Pretoria and the National Research Foundation.

\section{REFERENCES}

ANYAN, J.J., SENEY, M.L., HOLLEY, A., BENGSTON, L., GOLDMAN, B.D., FORGER, N.G. \& HOLMES, M.M. 2011. Social status and sex effects on neural morphology in Damaraland mole-rats, Fukomys damarensis. Brain Behavior and Evolution 77: 291-298.

BENNETT, N.C. 1990. Behavior and social-organization in a colony of the Damaraland mole-rat Cryptomys damarensis. Journal of Zoology, London 220: 225-248.

BENNETT, N.C. 1994. Reproductive suppression in social Cryptomys damarensis colonies - a lifetime of socially-induced sterility in males and females (Rodentia, Bathyergidae). Journal of Zoology, London 234: 25-39.

BENNETT, N.C. 2011. Teasing apart socially-induced infertility in non-reproductive female Damaraland mole-rats, Fukomys damarensis (Rodentia: Bathyergidae). Integrative Zoology 6: 311-320.

BENNETT, N.C. \& FAULKES, C.G. 2000. African Mole-rats: Ecology and Eusociality. Cambridge University Press, Cambridge.

BENNETT, N.C., FAULKES, C.G. \& MOLTENO, A.J. 1996. Reproductive suppression in subordinate, non-breeding female Damaraland mole-rats: two components to a lifetime of socially induced infertility. Proceedings of the Royal Society B - Biological Sciences 263: 1599-1603. 
BENNETT, N.C. \& JARVIS, J.U.M. 1988. The socialstructure and reproductive-biology of colonies of the mole-rat, Cryptomys damarensis (Rodentia, Bathyergidae). Journal of Mammalogy 69: 293-302.

BENNETT, N.C., JARVIS, J.U.M., AGUILAR, G.H. \& McDAID, E.J. 1991. Growth and Development in 6 species of African mole-rats (Rodentia, Bathyergidae). Journal of Zoology, London 225: 13-26.

BENNETT, N.C., JARVIS, J.U.M., FAULKES, C.G. \& MILLAR, R.P. 1993. LH responses to single doses of exogenous GnRH by freshly captured Damaraland mole-rats, Cryptomys damarensis. Journal of Reproduction and Fertility 99: 81-86.

BENNETT, N.C., JARVIS, J.U.M., MILLAR, R.P., SASANO, H. \& NTSHINGA, K.V. 1994. Reproductive suppression in eusocial Cryptomys damarensis colonies - socially-induced infertility in females. Journal of Zoology, London 233: 617-630.

BUFFENSTEIN, R. 2008. Negligible senescence in the longest living rodent, the naked mole-rat: insights from a successfully aging species. Journal of Comparative Physiology B - Biochemical Systemic and Environmental Physiology 178: 439-445.

COONEY, R. \& BENNETT, N.C. 2000. Inbreeding avoidance and reproductive skew in a cooperative mammal. Proceedings of the Royal Society B - Biological Sciences 267: 801-806.

COX, R.M. \& CALSBEEK, R. 2010. Severe costs of reproduction persist in anolis lizards despite the evolution of a single-egg clutch. Evolution 64: 1321-1330.

DAMMANN, P. \& BURDA, H. 2006. Sexual activity and reproduction delay ageing in a mammal. Current Biology 16: R117-R118.

DAMMANN, P., SELL, D.R., BEGALL, S., STRAUCH, C. \& MONNIER, V.M. 2012. Advanced glycation end-products as markers of aging and longevity in the long-lived Ansell's mole-rat (Fukomys anselli). Journals of Gerontology Series a - Biological Sciences and Medical Sciences 67: 573-583.

DAMMANN, P., SUMBERA, R., MASSMANN, C., SCHERAG, A. \& BURDA, H. 2011. Extended longevity of reproductives appears to be common in Fukomys mole-rats (Rodentia, Bathyergidae). PLoS One 6: e18757

FAULKES, C.G. \& BENNETT, N.C. 2001. Family values: group dynamics and social control of reproduction in African mole-rats. Trends in Ecology and Evolution 16: 184-190.

FLATT, T. 2011. Survival costs of reproduction in Drosophila. Experimental Gerontology 46: 369-375.

HATLE, J.D., PATERSON, C.S., JAWAID, I., LENTZ, C., WELLS, S.M. \& FRONSTIN, R.B. 2008. Protein accumulation underlying lifespan extension via ovariectomy in grasshoppers is consistent with the disposable soma hypothesis but is not due to dietary restriction. Experimental Gerontology 43: 900-908.

HEINZE, J. \& SCHREMPF, A. 2008. Aging and reproduction in social insects - a mini-review. Gerontology 54: 160-167.
JARVIS, J.U.M. 1981. Eusociality in a mammal - cooperative breeding in naked mole-rat colonies. Science 212: 571-573.

JARVIS, J.U.M. \& BENNETT, N.C. 1991. Ecology and behavior of the family Bathyergidae. In: The Biology of the Naked Mole-rat, (eds) P.W. Sherman, J.U.M. Jarvis \& R.D. Alexander, pp. 66-96. Princeton University Press, Princeton.

JARVIS, J.U.M. \& BENNETT, N.C. 1993. Eusociality has evolved independently in 2 genera of Bathyergid mole-rats - but occurs in no other subterranean mammal. Behavioral Ecology and Sociobiology 33: 253-260.

KELLER, L. \& GENOUD, M. 1997. Extraordinary lifespans in ants: a test of evolutionary theories of ageing. Nature 389: 958-960.

KIRKWOOD, T.B.L. \& ROSE, M.R. 1991. Evolution of senescence-late survival sacrificed for reproduction. Philosophical Transactions of the Royal Society of London Series B - Biological Sciences 332: 15-24.

LOVEGROVE, B.G. 1988. Colony size and structure, activity patterns and foraging behavior of a colony of the social mole-rat Cryptomys damarensis (Bathyergidae). Journal of Zoology, London 216: 391-402.

MOLTENO, A.J. \& BENNETT, N.C. 2000. Anovulation in non-reproductive female Damaraland mole-rats (Cryptomys damarensis). Journal of Reproduction and Fertility 119: 35-41.

NOORDWIJK, A.J.V. \& JONG, G.D. 1986. Acquisition and allocation of resources: their influence on variation in life history tactics. American Naturalist 128: 137-142.

READ, A.F. \& HARVEY, P.H. 1989. Life-history differences among the eutherian radiations. Journal of Zoology, London 219: 329-353.

REMOLINA, S.C. \& HUGHES, K.A. 2008. Evolution and mechanisms of long life and high fertility in queen honey bees. Age 30: 177-185.

RICKLEFS, R.E. \& WIKELSKI, M. 2002. The physiology/life-history nexus. Trends in Ecology \& Evolution 17: 462-468.

ROFF, D.A. 1992. The evolution of Life Histories: Theory and Analysis. Chapman and Hall, New York.

SHERMAN, P.W. \& JARVIS, J.U.M. 2002. Extraordinary life spans of naked mole-rats (Heterocephalus glaber). Journal of Zoology, London 258: 307-311.

SPEAKMAN, J.R. 2008. The physiological costs of reproduction in small mammals. Philosophical Transactions of the Royal Society B - Biological Sciences 363:375-398.

STEARNS, S.C. 1989. Trade-offs in life-history evolution. Functional Ecology 3: 259-268.

STEARNS, S.C. 1992. The Evolution of Life Histories. Oxford University Press, Oxford.

WILLIAMS, G.C. 1966. Natural selection, the costs of reproduction, and a refinement of Lack's principle. American Naturalist 100: 687-690.

YOUNG, A.J. \& BENNETT, N.C. 2010. Morphological divergence of breeders and helpers in wild Damaraland mole-rat societies. Evolution 64: 3190-3197.

Responsible Editor: P.J. Taylor 